\title{
Application du plasma atmosphérique froid en oncologie : une revue systématique
}

\author{
A. Dubuc (Toulouse), P. Monsarrat (Toulouse), S. Laurencin-Dalicieux (Toulouse), F. \\ Virard (Lyon), J.P. Sarrette (Toulouse), N. Merbahi (Toulouse), S. Cousty (Toulouse)
}

Introduction : Le plasma atmosphérique froid est un gaz ionisé produit à pression atmosphérique. Plusieurs applications médicales sont étudiées, notamment la cicatrisation des plaies chroniques et l'effet antimicrobien. En effet, le traitement par plasma permet de générer de nombreuses espèces réactives de l'oxygène et de l'azote. L'application d'un tel traitement in-vitro sur des cellules eucaryotes a montré de nombreux effets cellulaires tel que l'apoptose. Les applications dans le domaine de l'oncologie ont par conséquent été étudiées. Objectif : L'objectif de cette revue systématique est d'analyser l'utilisation du plasma atmosphérique froid en oncologie ainsi que les méthodologies (lignées cellulaires ciblées, paramètres physiques, thérapies directes ou indirectes) mises en œuvre jusqu'à ce jour. Matériels et méthodes : Les bases de données Pubmed, ICTRP et Google Scholar ont été explorées jusqu'au 17/01/2017 afin de recenser les études traitant de l'utilisation du plasma en oncologie, que ce soit des études in-vitro, in-vivo ou des essais cliniques. Résultats : 150 articles originaux ont été inclus. Les Jets de plasma sont les systèmes de production de plasma les plus utilisés (73,3\%). L'hélium est le gaz le plus utilisé (34\%) suivi par l'air (28\%) et l'argon (19,3\%). Les études sont principalement in-vitro (94\%). L'application directe du plasma est la plus représentée $(84,2 \%)$. Les lignées cellulaires ciblées sont la plupart dérivées de lignées cancéreuses humaines (82\%), en particulier des lignées issues de cancer du cerveau (16,6\%). Conclusions : Cette étude met en évidence la multiplicité de moyens de production et d'applications clinques du plasma atmosphérique froid en oncologie. Alors que certains dispositifs peuvent être utilisés directement sur les patients, d'autres ouvrent la voie au développement de nouveaux produits pharmaceutiques qui pourraient être produits à échelle industrielle. L'utilisation clinique du plasma nécessite la mise au point de protocoles fiables et standardisés afin de déterminer le plasma le plus adapté à chaque type de cancers et d'envisager son association avec les traitements conventionnels. 